\title{
DRIFT DIFFUSION MODELLING REVEALS DECISION MECHANISMS UNDERLYING CONSUMERS' EVALUATION OF PRICES.
}

\author{
Jasper Dezwaef ${ }^{1,+}$, Silvia Formica ${ }^{1,{ }^{\dagger}}$, Emiel Cracco $^{1}$, Pieter Huycke ${ }^{1}$, Jelle Demanet ${ }^{1}$, Timothy Desmet $^{2,3,4}$ and \\ Marcel Brass ${ }^{1}$ \\ ${ }^{1}$ Department of Experimental Psychology, Ghent University, Ghent, Belgium \\ ${ }^{2}$ Faculty of Economic and Social Sciences, Vrije Universiteit Brussel, Brussels, Belgium \\ ${ }^{3}$ Solvay Business School, Brussels, Belgium \\ ${ }^{4}$ Hult International Business School, UG Campus London, London, United Kingdom \\ ${ }^{\dagger}$ These authors contributed equally to this paper
}

Declaration of Interest: none

Acknowledgements: This work was funded by VLAIO (IWT150721) in the form of a Baekeland mandate awarded to Jasper Dezwaef.

Corresponding author: Jasper Dezwaef (j.dezwaef@gmail.com) 


\section{ABSTRACT}

Little is known about how price evaluation processes unfold. In the current study we explored if reaction times (RTs) can be used to study price evaluations. Additionally, we explored to what extent drift diffusion models (DDMs) are suitable to decompose the different aspects that underlay this decision processes. In a behavioral experiment, participants were asked to evaluate prices as fast as possible as 'cheap' or 'expensive'. We expected that the time needed to evaluate prices would vary in accordance with a price manipulation that was used, and that RTs therefore could be interpreted a proxy of decision difficulty. Analysis of the behavioral data provided evidence for this hypothesis: very cheap and very expensive prices were evaluated faster compared to ambiguous prices. Then, drift diffusion models (DDMs) were used to decompose the different aspect of this decision process, with the goal to obtain a more fine-grained understanding of how the effect in RT data emerged. Results showed that the drift rate of the model was modulated by the price manipulation. Whereas there was no significant effect of the price manipulation on the nondecision time and the starting point parameter. We then contrasted the findings of the RT analysis with the results of the DDMs and outlined what the added value of DDMs is within this context.

\section{INTRODUCTION}

Consumers encounter and evaluate numerous prices every day. Ample evidence suggests that during the evaluation of selling prices, internal reference prices (IRPs) are used 
as subjective threshold values to which selling prices are compared (Mayhew \& Winer, 1992; Monroe, 1973; Thomas \& Menon, 2007; Winer, 1986). These IPRs are constructed based on prior purchase experiences, stored in memory, and retrieved when needed (Mazumdar, Raj, \& Sinha, 2005). Furthermore, IRPs are flexible constructs that can vary depending on the context (Adaval \& Monroe, 2002; Kalyanaram \& Winer, 1995; Mayhew \& Winer, 1992). However, because traditional economic studies have mainly been concerned with behaviors that result out of decisions (Clithero, 2018), little is known about specific cognitive mechanisms related to the use of IRPs as an evaluation threshold for decision making.

Reaction times (RTs) are one of the most commonly used dependent variables to study these cognitive mechanisms (e.g. Cohen, Dunbar, \& Mcclelland, 1989; Ratcliff \& Rouder, 1998). RTs capture the time elapsed between the presentation of a stimulus and the reaction of the participant, and are often interpreted as a proxy of decision difficulty (Bell, Loomis, \& Cervone, 1982; Wright \& Ayton, 1988). Implying that high RTs reflect a difficult task, whereas low RTs reflect an easy task. However, RTs do not provide information about the different decision processes that contributed to the formation of the decision. RTs only the represent total time needed to make a decision. To address this issue, Drift Diffusion Model (DDMs) are often used to integrate information of both RTs and binary responses in simple binary decision tasks, allowing to investigate the subprocesses that underlying these decisions (Voss, Nagler \& Lerche, 2013). Initially, DDM studies modelled RT data of simple perceptual decisions (Bodur, Klein, \& Arora, 2016; Heekeren, Marrett, \& Ungerleider, 2008; Philiastides, Ratcliff, \& Sajda, 2006), while more recent work successfully used DDMs to explain valuebased decisions (Gold \& Shadlen, 2007; Krajbich, Armel, \& Rangel, 2010; Mormann, Malmaud, Huth, Koch, \& Rangel, 2010). 
The underlying assumption of the DDM framework is that when participants are faced with a binary choice, the evidence is steadily accumulated towards one of the two options. When enough evidence has been accumulated to reach one of the two decision boundaries, the decision is made, and the accumulation process ends. Therefore, the advantage of applying diffusion models to explain decision behavior is twofold. First, DDMs consider the whole distribution of RTs for both choice options on a trial-by-trail basis, therefore maximizing the usage of available information (Ratcliff \& Rouder, 1998). Second, and crucially, DDMs provide more fine-grained information about specific aspects of decision making and, furthermore, integrate speed-accuracy trade-offs (Voss, Rothermund, \& Voss, 2004).

In its most basic formulation, the DDM estimates four parameters. The drift rate $(v)$ is the average slope of the diffusion process and describes the speed of information accumulation. Faster drift rates lead to faster RTs, whereas if the process of information accumulation is slow or inefficient, RTs become slower. Boundary separation (a) represents the distance between the two decision thresholds and thus the amount of information that is needed to reach one of them. This value plays a role in determining both the timing that it takes to achieve a decision and its accuracy, reflecting speed-accuracy trade-off in more conservative or liberal decision styles. When the boundaries are very distant, more evidence needs to be accumulated to reach the decision threshold, resulting in slower RTs but also in lower error rates as it becomes more unlikely to cross the wrong decision threshold accidentally. The information accumulation process originates from the starting point $(z)$, which is usually placed in between the two options $(a / 2)$. Shifts in the starting point reflect a bias towards one of the two options. For example, one can be more prone to give one specific response if it leads to a greater reward concerning the alternative. Lastly, the non-decision time (t0), accounts for all the non-decision related processes that occur prior or after the 
evidence accumulation process. For instance, it accounts for the latency due to stimulus encoding, retrieval of information from memory, and motor response execution. Importantly, the non-decision time does not affect the accumulation process, as it reflects processes occurring before and after the actual decision. Therefore, higher values of t0 lead to a shift in the RTs distribution, without modifying its shape.

In the current study, participants were presented with product-price combinations and then asked to evaluate them as fast as possible as 'cheap' or 'expensive'. Crucially, the magnitude of the prices was manipulated to be perceived as ambiguous (i.e., close to expected IRP) or non-ambiguous (i.e., distant from the assumed IRP). This manipulation made it possible to test two hypotheses. First, we expected that ambiguous prices would result in slower price evaluations compared to unambiguous prices. This effect was observed in our earlier work and is assumed to provide information about consumers' IRPs (Dezwaef, Cracco, Demanet, Desmet, \& Brass, 2019; Dezwaef et al., 2020). That is, if prices are neither clearly 'cheap' nor 'expensive', more conflict between two response options should be experienced and responses should be slower. Secondly, and more importantly, we investigated if DDM parameters were modulated by this effect, hence we attempted to decompose the different aspects of the decision processes that guide price evaluation. Foremost, we expected the drift rate $(v)$ to be influenced by the ambiguity of the price. Based on the results of previous work (Dezwaef et al., 2019, 2020) we expected that more ambiguous prices would result in lower drift rates, as more evidence would need to be accumulated to decide between 'cheap' or 'expensive'. However, it is also possible that differences in RTs are not related to the ambiguity of the price but instead to the retrieval of the IRP. Then, we would expect to see variations in the non-decision time (to) depending on the price manipulation. Moreover, it is also plausible to assume that respondents might have the tendency to evaluate prices more 
as expensive than as cheap. That is, it might be a default mechanism that is used by consumers to label prices as expensive. If there is such a bias, then this should be picked up by the starting point (z) parameter. Finally, because boundary separation (a) reflects the caution at which responses are given, we used interindividual differences in this parameter as a measure of impulsivity and investigated to what extent this parameter influences price evaluation. That is, some individuals might be more impulsive when they evaluate prices, resulting in faster reaction times, compared to people that are less impulsive. Therefore, the boundary separation (a) parameter can provide useful information about differences that are not directly related to the price that had to be evaluated but rather reflect general interindividual differences.

\section{METHODS}

\section{Participants}

The experiment was completed by 34 participants with normal or corrected-to-normal vision (19 females; MAge $=21.6$ years, SDAge $=3.2$ ) who were recruited via the online recruitment platform of the University of Ghent. The study was conducted in accordance with the ethical standards of the 1964 Declaration of Helsinki and approved by the Institutional Review Board from the Faculty of Psychology and Educational Science of Ghent University. Prior to the experiment, participants gave their written informed consent and were informed that participation was voluntary, and that all data would be processed and stored anonymously. 


\section{Procedure \& Materials}

Participants were briefly introduced to the task and invited to take place in front of the computer. Further instructions on the screen asked participants to evaluate a series of product-price combinations as 'cheap' or 'expensive' by pressing on the corresponding button of the keyboard ('f-button' = cheap; ' $\mathrm{j}$-button' = expensive). Participants were instructed to do this as fast as possible and not to overthink their evaluations. However, to reassure that each participant was actually responding fast, an adaptive response deadline (ADR) was implemented. This implied the response deadline was adapted continuously throughout the experiment, based on the reaction times of the participant on the previous trials. To calculate the ADR, the average and standard deviation of the previous eight trials were computed. Then, to calculate the actual ADR for the current trial, 1.5 SD was added to the calculated mean. No response deadline was implemented on the first eight trials.

In total, eight products (iced tea, cola, cream cheese, mints, orange juice, potato chips, ham and candy) of well-known brands were selected for the study. Based on the selling price of these products at the moment of the study (i.e. retail price), additional price stimuli ('cheap', 'expensive' or 'ambiguous') were constructed. Specifically, we either added or subtracted $10 \%, 40 \%$ or $70 \%$ to the retail price. We expected that the degree of ambiguity regarding of the price evaluation would decrease as the proportion of the manipulation increased. In the end, the lowest price used was $€ 0.37$ and the highest price used was $€ 6.36$. In total, this resulted in 56 product-price combinations that were presented six times in random order, equally divided across six blocks.

Stimulus presentation was controlled with the PsychoPy package (Peirce, 2009), a Python library used to run cognitive experiments. Each trial started with a fixation cross that was presented on the screen for 500 milliseconds (see figure 1). After the fixation cross, the 
product was presented 2500 milliseconds. Then the product was removed from the screen and the price stimulus was presented for duration of the ADR or until a response was given. The warning message with "Te traag!" (i.e., "Too slow" in Dutch) appeared on the screen for $500 \mathrm{~ms}$ if participants were too slow. The inter-trial interval was set to 1000 milliseconds. The experimental procedure lasted approximately 30 minutes.

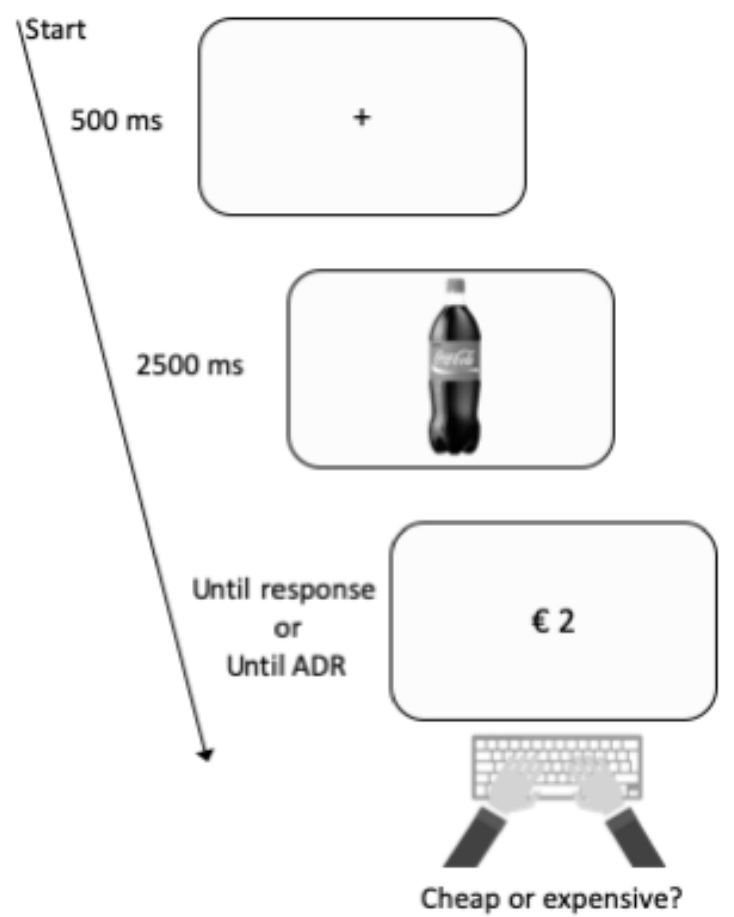

Figure 1. Trial sequence used during the experiment. First, participants were presented a fixation cross for 500 milliseconds. Then, the cross was replaced by a product, which was presented for 2500 milliseconds. Finally, the product was replaced by the price and the participant was obliged to evaluate the price as fast as possible. When the adaptive response deadline was exceeded, the experiment continued, and the participant was instructed to respond faster on the next trial. 


\section{Data analysis}

\section{Data preprocessing and RT analysis}

The raw data was prepared for analysis in RStudio (R Core Team, 2018). Prior to further analysis, trials exceeding the adaptive response deadline were removed from the analysis (4.8\%). Then we further removed outliers by excluding RTs that were 1.5SD above or below the average RT of the participant (8.0\%). Quadratic mixed effects models (Baayen, Davidson, \& Bates, 2008) were used to investigate the relationship between RTs and price ambiguity. The quadratic term of price condition was included as numerical predictor (i.e., $\pm 70 \%, \pm 40 \%$, $\pm 10 \%$ and retail) in the models. By-participant and by-product random intercepts were included in order to control for interindividual and interproduct differences. The optimal random slopes structure was determined using a backwards selection procedure (Matuschek, Kliegl, Vasishth, Baayen, \& Bates, 2017). The 'Ime4' package (Bates, Mächler, Bolker, \& Walker, 2014) in R (R Core Team, 2018) was used to fit the models. P-values were calculated using Satterthwaite approximated degrees of freedom and as measure of effect size measures, the $\mathrm{R}^{2}$ statistic of the model was computed using the R library 'MuMIn' (Barton, 2018).

\section{Drift Diffusion Model}

To fit the data used for the RT analysis, we used the hierarchical drift diffusion model (HDDM) (Wiecki, Sofer, \& Frank, 2013). The HDDM is considered hierarchical because it first uses data from all the participants to estimate group-level mean parameters, and then it uses these group-level priors to constrain the estimation of the subject-specific parameters. This approach allows for more stable results, even with fewer data per participant with respect to the traditionally used algorithms (Lerche, Voss, \& Nagler, 2017). 
The estimation of model parameters distributions within the HDDM toolbox relies on a Markov-chain Monte Carlo sampling procedure (Gamerman \& Lopes, 2006). We used a chain with 10000 samples; the first 1000 samples were discarded as burn-in, to allow for the sampling procedure to settle around a value after an initial more exploratory sampling. To reduce autocorrelation in the retained samples, we additionally discarded every second sample. Finally, we fitted our data with 'cheap' and 'expensive' as response boundaries using the hddm.HDDM() function of the toolbox (Wiecki et al., 2013). Given that we were interested in investigating how the parameters were affected by our price manipulation, we treated the 7 price levels as different conditions ('retail', '-10\%, '-40\%, '-70\%, '+10\%, '+40\%, '+70\%).

Since we hypothesized our price manipulation to be reflected on either different drift rates or non-decision time between conditions, we ran models with different combinations of these parameters set free to vary between conditions (namely, only v, only t0, or both). We then compared them by means of the Deviance Information Criterion (DIC): the lower its value, the better the model explains the data. Notably, this measure is known to be slightly biased in favor of more complex models, but we assumed that very large differences in DIC values would indicate a real advantage of the model. In all models, we also estimated the $z$ value for each subject, to investigate possible inter-individual biases towards one of the two responses. Moreover, we instructed the model fitting process to tolerate up to $5 \%$ of outliers in the dataset (p_outlier $=0.05)$.

The chain and its autocorrelation trace were visually inspected to confirm successful convergence. Moreover, Gelman-Rubin R hat statistics were computed to evaluate withinand between-chain variance. Parameters with an $\mathrm{R}$ hat between 0.99 and 1.01 were deemed to be successfully converged (Gelman \& Rubin, 1992). Finally, as an additional measure of 
goodness of fit, we simulated data from the parameters estimated by the model and compared the simulated distribution with the observed data.

Being constrained by group-priors, the subject-level parameters estimated by the HDDM violate the independency assumption (i.e., the subject-level parameters are drawn from the same distribution) and thus cannot be analyzed with frequentist statistics. Instead, parameter differences between conditions can be quantified by looking at the percentage of overlap of their posterior distributions. For each modeled parameter, the toolbox output consists of a gaussian distribution for each experimental condition. The further apart these distributions are, the greater is the difference between the conditions captured by the specific parameter. Measuring the proportion of overlap between distributions allows to finely assess how far apart, and thus different, they are. For example, a high probability of a given parameter having a higher value in Condition 1 compared to Condition 2 would result in a small posterior distributions overlap, because the two distributions would be very far apart from each other in the parameter space and only minorly overlap. To make these results more easily interpretable, we considered differences in posterior distributions above $95 \%$ as strong evidence in favor of a difference between conditions.

To investigate further the relationship between the boundary separation parameter (a) and RTs, an additional factor was created. Based on the mean a value, a split-half procedure was applied to create the factor impulsivity with two levels (high a vs low a). The rationale behind this approach is to group participants based on the $a$ parameter, which is assumed to capture the decisional approach adopted by the individual in the context of price evaluation. A high value of a reflects distant boundary and thus a cautious decision style, whereas low values of a imply faster but also more impulsive responses. 


\section{RESULTS}

\section{RT analysis}

A quadratic mixed analysis was performed to test the relationship between RTs and price condition. The results revealed a statistically significant quadratic effect of price condition, $\left(t(10428)=-17.10, R^{2}=.47, p<.001\right)$, indicating that RTs followed a reversed $U$ shaped pattern and that the time needed to evaluate prices depended on the perceived price ambiguity. Figure 2 shows both the observed data points and the model predictions. As can be seen, the slowest RTs were observed when the prices were assumed to be most ambiguous. On the contrary, the linear effect was not significant, $\left(t(10429)=1.09, R^{2}=.47, p\right.$ $=.27)$

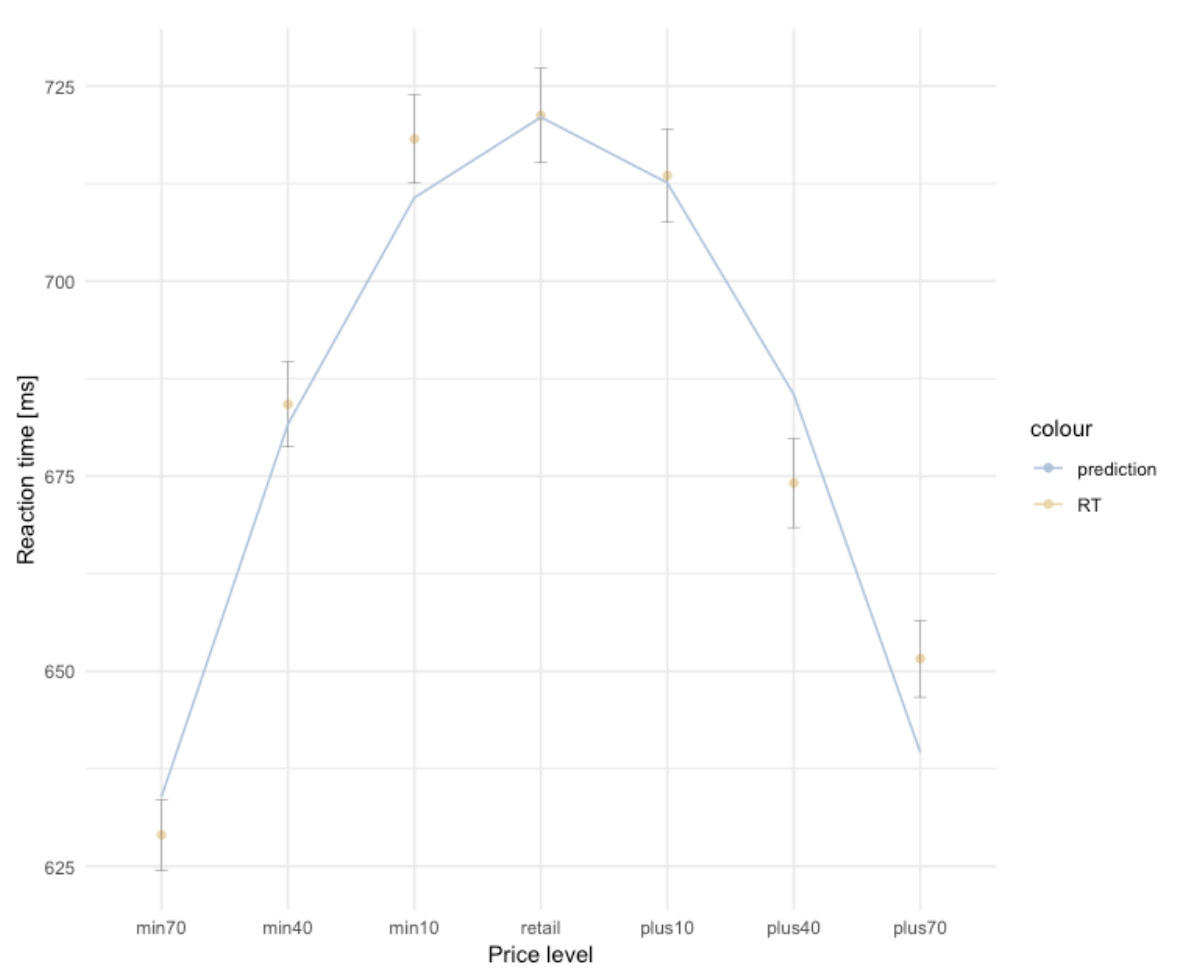

Figure 1. RTs varied in accordance with the price manipulation. More ambiguous prices resulted in faster price evaluations, whereas ambiguous prices were evaluated significantly slower. The blue line represents the model prediction and the yellow dots represent the observed data. 


\section{Drift Diffusion Model}

We fitted different models, allowing in each of them a different set of parameters to vary freely between conditions (only $v$, only t0, both $v$ and t0). Model comparison revealed that the model in which both drift rate and non-decision time were set to be dependent on the price manipulation provided a substantially better fit compared with drift rate alone $(\triangle D I C$ $=-325)$ or non-decision time alone $(\Delta \mathrm{DIC}=-4,595)$. All measures of goodness of fit confirmed successful parameter estimation by our winning model. Therefore, we analyzed the parameters estimated from this model.

\section{Starting point}

First, to check for possible biases towards one of the two possible responses, we estimated the starting point of the decision process for each subject. If both alternative responses are equally likely to be chosen before the onset of the target, the starting point should be at the half point between the two boundaries $(a / 2$, which is conventionally normalized to 0.5$)$. Overall, participants showed no biased preference for either of the two options ( $M=0.51, S D=0.13)$, confirming the reliability of our price manipulation.

\section{Drift rate}

The drift rate showed to be sensitive to the price level manipulation. Negative values here refer to drift rates pointing towards the lower boundary, whereas conditions in which the response associated with the upper boundary was more frequent have a positive drift rate. Crucially, drift rate magnitude increased linearly with the degree of price manipulation (Table 1). 


\begin{tabular}{|c|c|}
\hline PRICE COND & $M(S D)$ \\
\hline Retail & $0.20(0.43)$ \\
\hline$-10 \%$ & $-0.14(0.44)$ \\
\hline$-40 \%$ & $-1.35(0.63)$ \\
\hline$-70 \%$ & $-2.33(0.95)$ \\
\hline$+10 \%$ & $0.50(0.51)$ \\
\hline$+40 \%$ & $1.20(0.61)$ \\
\hline$+70 \%$ & $1.86(0.88)$ \\
\hline
\end{tabular}

Table 1. The mean and standard deviations of the drift rates (z), for each price conditions, as estimated by our model.

The less ambiguous the presented price was, the faster the evidence accumulation process progressed towards the corresponding boundary. Posterior distributions comparison provided strong evidence in favor of a difference in drift rate between extreme $( \pm 70 \%)$ and medium ( $\pm 40 \%$ ) price manipulations. Analogously, drift rate values could effectively capture the differences between medium $( \pm 40 \%)$ and ambiguous $( \pm 10 \%)$ price manipulations. The conditions showing the highest proportion of posterior distributions overlap were the highly ambiguous prices $( \pm 10 \%)$ and retail price, suggesting that the decision process in these conditions is more similar than in the other conditions (table 2).

\begin{tabular}{c|c} 
Comparison & Posterior probabilities differences \\
\hline$P(\min 40>\min 70)$ & 1.0 \\
$P(\min 10>\min 40)$ & 1.0 \\
$P($ retail $>\min 10)$ & 0.96 \\
$P($ plus $10>$ retail $)$ & 0.93 \\
$P($ plus $40>$ plus 10$)$ & 0.99 \\
$P($ plus $70>$ plus 40$)$ & 0.99
\end{tabular}

Table 2. Differences between price levels in the posterior probability distributions of the drift rate (z). 


\section{Non-decision time}

The non-decision parameter resulted to be not affected by the price manipulation (Table 3). Posterior distributions for all conditions overlapped, and statistical comparisons showed no difference between neighboring price manipulation. This pattern suggests that all the cognitive and motor processes involved in the task but not attributable to the decision process itself, among which the most relevant we assume to be the retrieval of the IRP, were not modulated by our manipulation (table 4).

\begin{tabular}{|c|c|}
\hline Price condition & $M / S D$ \\
\hline Retail price & $0.40(0.06)$ \\
\hline $\operatorname{Min} 10$ & $0.38(0.06)$ \\
\hline Min40 & $0.38(0.06)$ \\
\hline Min70 & $0.39(0.06)$ \\
\hline Plus10 & $0.38(0.05)$ \\
\hline Plus40 & $0.37(0.04)$ \\
\hline Plus70 & $0.39(0.06)$ \\
\hline
\end{tabular}

Table 3. The mean and standard deviations of the non-decision time (t0), for each price conditions, as estimated by our model.

\begin{tabular}{c|c} 
Comparison & Posterior probabilities differences \\
\hline$P(\min 40>\min 70)$ & 0.34 \\
$P(\min 10>\min 40)$ & 0.48 \\
$P($ retail $>\min 10)$ & 0.66 \\
$P($ plus $10>$ retail $)$ & 0.25 \\
$P($ plus $40>$ plus 10 $)$ & 0.46 \\
$P($ plus $70>$ plus 40$)$ & 0.71
\end{tabular}

Table 4. Differences between price levels in the posterior probability distributions of the non-decision time (t0) 


\section{Boundary separation as a measure of impulsivity}

A quadratic mixed analysis was performed to investigate the effect of price condition and impulsivity on RTs. The model included price condition (i.e., $\pm 70 \%, \pm 40 \%, \pm 10 \%$ and retail) as numerical predictor and impulsivity (high a vs. low a) as fixed effect. Again, by-participant and by-product random intercepts and random slopes for the numerical predictor price condition were added to the model. The main effect of impulsivity was statistically significant, $\left(t(32)=5.44, R^{2}=.47, p<.001\right)$, implying that participants with high a values respond slower compared to participants with low a values. The quadratic effect of price condition was again statistically significant, $\left(\mathrm{t}(10426)=-17.11, \mathrm{R}^{2}=.47, \mathrm{p}<.001\right)$, indicating that the RTs changed in accordance with the ambiguity of the price that had to be evaluated. However, the linear effect of price condition was not statically significant $\left(t(228)=1.17, R^{2}=.47, p<.24\right)$. Furthermore, the interaction between the quadratic term of price condition and impulsivity was also statistically significant $(\mathrm{t}(10425)=-10.36, \beta=-2014.3, \beta S E=194.5, p<.001)$, suggesting that the effect of price condition on RTs is more pronounced for participants that are expected to be less impulsive. 


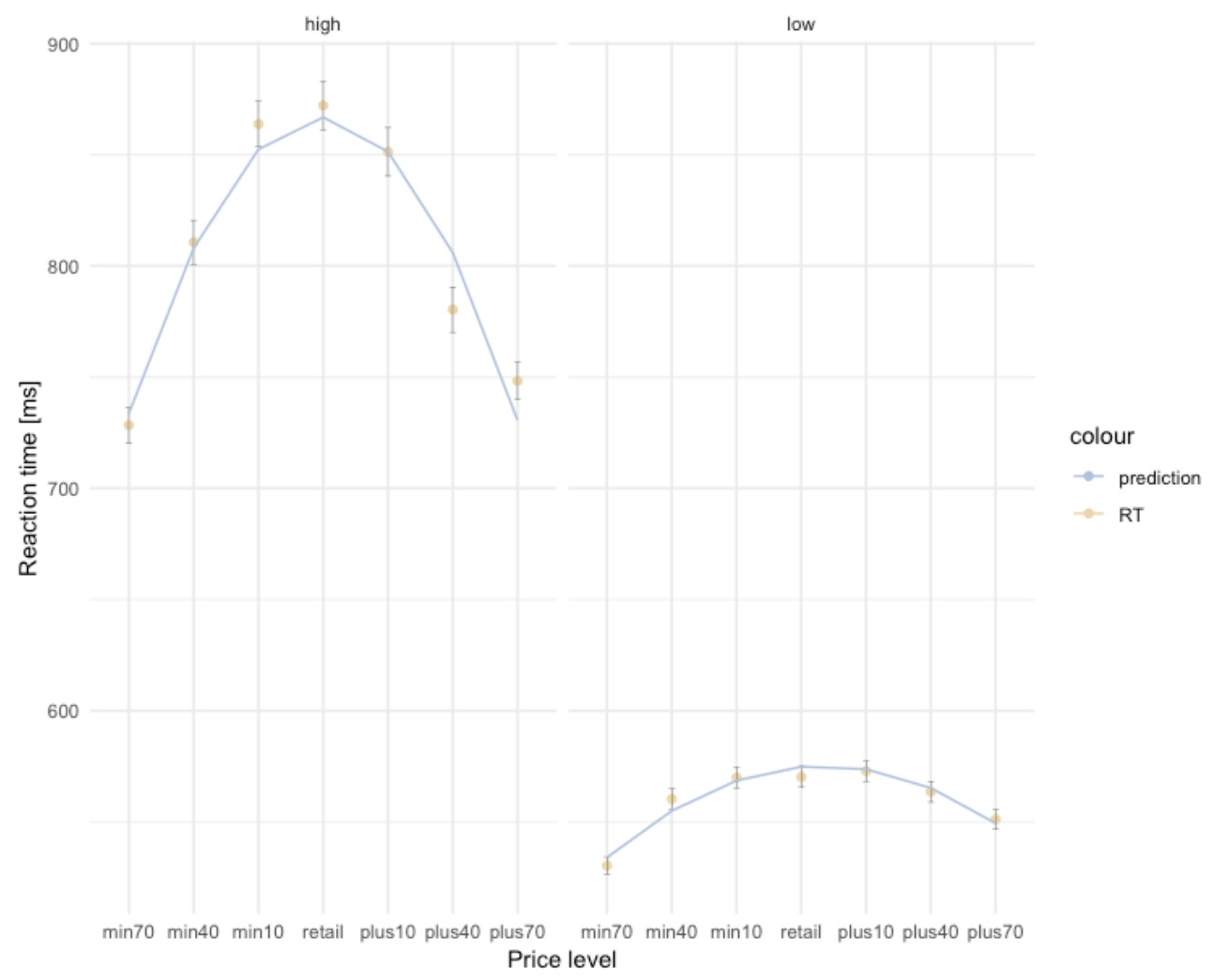

Figure 2. The effect of price condition as a function of impulsivity.

\section{DISCUSSION}

Internal reference prices (IRPs) are used by consumers to evaluate selling prices (Chen, 2009; Mayhew \& Winer, 1992). In the current study, we investigated whether RTs can provide information about IRPs and whether drift diffusion models (DDMs) can decompose the aspects of the decision process involved in price evaluations. To this end, we conducted an RT experiment in which participants had to evaluate prices as fast as possible. Crucially, the magnitude of the used price stimuli was manipulated. This manipulation created a context in which selling prices could be considered ambiguous, or not, by the participants. We expected that RTs would be informative about the magnitude of the internal reference price. That is, when prices are closely aligned with the IRP, RTs were expected to be slower because conflict would emerge between the responses. 
Mixed models were used to investigate the relationship between price ambiguity and RTs. The data exhibited the expected inverted U-shaped pattern (see figure 1), reflected in a statistically significant main effect of price condition ( $\pm 70 \%, \pm 40 \%, \pm 10 \%$, and retail). This evidence shows that it takes longer to evaluate a price as 'cheap' or 'expensive' if it is closely aligned with the IRP (i.e., $\pm 10 \%$ and retail price conditions). This effect is likely to be the result of response conflict (Botvinick, Braver, Barch, Carter, \& Cohen, 2001). Ample research shows that when the decision between two response becomes more difficult, RTs become slower (e.g., Bell et al., 1982). In this study, the closer the presented selling price was to the assumed IRP, the slower the observed RTs were. This finding replicates an effect that was reported in our previous work (Dezwaef et al., 2020). One possible explanation for this finding is that it is more challenging for participants to evaluate prices of the $\pm 10 \%$ or retail price conditions because the decision is more difficult. This would imply that more considerations or computations are needed before a decision can be made if the price differs only little from the threshold that is used to guide the evaluation process. Therefore, this evidence seems to suggest that RTs can provide valuable information about consumers' IRP. Because RTs alone do not allow to differentiate the aspects of the decision process required to make price evaluations, we also investigated whether DDMs can be used to provide a more fine-grained explanation of this effect.

Only the drift rate (v) was significantly modulated by price manipulation. As prices became more ambiguous (i.e., closer to the assumed IRP), the magnitude of the drift rate decreased, suggesting that more evidence had to be accumulated before the decision threshold was reached compared to when prices were clearly different from the IRP. Hence, one way to look at this finding is that the drift rate can be interpreted as the effort needed to compare the selling price with the IRP. The less clear the difference between both prices, the 
more evidence needs to be processed before the decision 'cheap' or 'expensive' is reached. Within this interpretation, the drift rate (v) can represent decision difficulty. That is, if prices are evidently 'cheap' or 'expensive' no real computations are required because it is clear that the price is deviant from the IRP. However, when this difference between the selling price and the IRP is less clear, the conflict between the two competing responses emerges, resulting in slower evidence accumulation and slower decision times. On the other hand, we found no evidence for the alternative hypothesis that the observed RT effects are the result of processes related to retrieving information related to the reference price from memory. However, the winning model required the inclusion of the non-decision (t0) parameter. An explanation for this finding might be that variance related to important processes, such as memory retrieval or motor execution, is captured by the parameter. However, these processes might be of little importance in the construction of price related decisions.

Just like the RT pattern, the drift rate might be useful to study how closely a tested price relates to the internal reference price of the consumer. For example, it might be the best approach to find the price with the highest $v$ value because it the underlying price evaluation appeared to be the most difficult according to the model. Other studies can then investigate how IRPs pinpointed by means of RTs relate to IRPs pinpointed by DDMs. Under the assumption that both RTs and the drift rate (v) are both tapping into the IRP, one could argue that the IRPs based on the analysis of the drift rate $(v)$ are more accurate since some processes that are reflected in RTs, such as memory retrieval processes, are not present in the drift rate.

Interestingly, the starting point $(z)$ parameter provided no evidence for the presence of a bias in the choice pattern. We anticipated to find a bias because we assumed that participants would rather be tending to evaluate prices as expensive. However, we did not 
find evidence for this hypothesis. One likely explanation is that there is no such bias and that participants were equally likely to evaluate prices as cheap or expensive before they saw the price. Another explanation would be that the applied time pressure in our experiment diminished the bias towards one of the two evaluations. Some studies showed that applying time pressure to decision making context can circumvent answering strategies (Ackermann \& Mathieu, 2015; Harrison \& Rutström, 2008).

Finally, we investigated to what extent the boundary separation (a) parameter varied between participants. The analysis showed that participants with a high a value had overall slower RTs. Possibly, the difference in this parameter reflects individual differences in the processing of price information. Some research on the effect of price promotions showed that individual differences can affect how consumers perceive promotions (e.g., Ortmeyer et al., 1991) and some studies showed that differences in impulsivity resulted in differences in consumer behavior (e.g., Huang \& Kuo, 2012). Hence, it is plausible that individual differences can explain the differences in the boundary separation parameter. For example, it could be put forward that individuals with high a values are less impulsive and more conservative when evaluating prices. This could also explain the observation that the effect in the RTs is more pronounced in this group, possibly indicating that they more carefully compare to the selling price of a product to their IRP. Regardless the exact interpretation, this finding is interesting because it shows that value generated by the DDM can be linked to modulations in the RTs. Hence, future research should further address this issue and control for traits such as impulsivity. 


\section{REFERENCES}

Ackermann, C., \& Mathieu, J. (2015). Implicit attitudes and their measurement: Theoretical foundations and use in consumer behavior research. Recherche et Applications En Marketing (English Edition), 30(2), 55-77.

Adaval, R., \& Monroe, K. B. B. (2002). Automatic Construction and Use of Contextual Information for Product and Price Evaluations. Journal of Consumer Research, 28(4), $572-588$.

Baayen, R. H., Davidson, D. J., \& Bates, D. M. (2008). Mixed-effects modeling with crossed random effects for subjects and items. Journal of Memory and Language, 59(4), 390412.

Bates, D., Mächler, M., Bolker, B. M., \& Walker, S. C. (2014). Fitting linear mixed-effects models using Ime4. Journal of Statistical Software, (67), 1-48.

Bell, P. A., Loomis, R. J., \& Cervone, J. C. (1982). Effects of Heat, Social Facilitation, Sex Differences, and Task Difficulty on Reaction Time. Human Factors, 24(1), 19-24.

Bodur, H. O., Klein, N. M., \& Arora, N. (2016). Online Price Search : Impact of Price Comparison Sites on Offline Price Evaluations. Journal of Retailing, 91(1), 125-139.

Botvinick, M. M., Braver, T. S., Barch, D. M., Carter, C. S., \& Cohen, J. D. (2001). Conflict Monitoring and Cognitive Control. Psychological Review, 108(3), 624-652.

Chen, C. Y. (2009). Who I am and how I think: The impact of self-construal on the roles of internal and external reference prices in price evaluations. Journal of Consumer Psychology, 19(3), 416-426.

Clithero, J. A. (2018). Response times in economics : Looking through the lens of sequential sampling models. Journal of Economic Psychology, 69, 61-86. 
Cohen, J. D., Dunbar, K., \& Mcclelland, J. L. (1989). On the control of automatic processes: a paralled distributed processing account of the stroop effect. Psychological Review, 97(3), 322.

Dezwaef, J., Cracco, E., Demanet, J., Desmet, T., \& Brass, M. (2019). Beyond asking: Exploring the use of automatic price evaluations to implicitly estimate consumers ' willingness-topay. PLOS ONE, 1-16.

Dezwaef, J., Dossche, W., Cracco, E., Demanet, J., Desmet, T., \& Brass, M. (2020). Prospecting the use of reaction times, response force and partial response force to estimate consumers' willingness-to-pay. PsyArXiv. https://doi.org/10.31234/osf.io/vkt2z

Gamerman, D., \& Lopes, H. F. (2006). Markov Chain Monte Carlo: Stochastic Simulation for Bayesian Inference, Second Edition. Texts in statistical science (Vol. 1). Taylor \& Francis. Gelman, A., \& Rubin, D. B. (1992). Inference from iterative simulation using multiple sequences. Statistical Science, 7(4), 457-472.

Gold, J. I., \& Shadlen, M. N. (2007). The Neural Basis of Decision Making. Annu Rev Neurosci, $30,535-574$.

Harrison, G. W., \& Rutström, E. (2008). Experimental Evidence on the Existence of Hypothetical Bias in Value Elicitation Methods.

Heekeren, H. R., Marrett, S., \& Ungerleider, L. G. (2008). The neural systems that mediate human perceptual decision making. Nat Rev Neurosci, 9.

Huang, Y., \& Kuo, F. (2012). Electronic Commerce Research and Applications How impulsivity affects consumer decision-making in e-commerce. Electronic Commerce Research and Applications, 11(6), 582-590.

Kalyanaram, G., \& Winer, R. S. (1995). Empirical Generalizations from Reference Price Research. Marketing Science, 14(3), 161-169. 
Krajbich, I., Armel, C., \& Rangel, A. (2010). Visual fixations and the computation and comparison of value in simple choice. Nature Neuroscience, 13(10), 1292-1298.

Lerche, V., Voss, A., \& Nagler, M. (2017). How many trials are required for parameter estimation in diffusion modeling? A comparison of different optimization criteria. Behavior Research Methods, 49(2), 513-537.

Matuschek, H., Kliegl, R., Vasishth, S., Baayen, H., \& Bates, D. (2017). Balancing Type I error and power in linear mixed models. Journal of Memory and Language, 94, 305-315.

Mayhew, G. E., \& Winer, R. S. (1992). An Empirical Analysis of Internal and External Reference Prices Using Scanner Data. Journal of Consumer Research, 19, 62-71.

Mazumdar, T., Raj, S. P., \& Sinha, I. (2005). Reference Price Research: Review and Propositions. Journal of Marketing, 69(4), 84-102.

Monroe, K. B. (1973). Buyers' Subjective Perceptions of Price. Journal of Marketing Research, 10(1), 70-80.

Mormann, M. M., Malmaud, J., Huth, A., Koch, C., \& Rangel, A. (2010). The Drift Diffusion Model Can Account for the Accuracy and Reaction Time of Value-Based Choices Under High and Low Time Pressure. Judgement and Decision Making, 5(6), 437-449.

Ortmeyer, G., Lattin, J. M., Montgomery, D. B., Ortmeyer, G., Lattin, J. M., \& Montgomery, D. B. (1991). Individual Differences in Response to Consumer Promotions. International Journal of Research in Marketing, 8(3), 169-186.

Peirce, J. W. (2009). Generating stimuli for neuroscience using PsychoPy. Frontiers in Neuroinformatics, 2(January), 1-8.

Philiastides, M. G., Ratcliff, R., \& Sajda, P. (2006). Neural Representation of Task Difficulty and Decision Making during Perceptual Categorization: A Timing Diagram. Journal of Neuroscience, 26(35), 8965-8975. 
Ratcliff, R., \& Rouder, J. N. (1998). Modeling response times for two-choice decisions. Psychological Science, 9(5), 347-356.

Thomas, M., \& Menon, G. (2007). When Internal Reference Prices and Price Expectations Diverge: The Role of Confidence. Journal of Marketing Research, 44(3), 401-409.

Voss, A., Nagler, M., Lerche, V., Institut, P., \& Heidelberg, R. (2013). Diffusion Models in Experimental Psychology A Practical Introduction, 60(6), 385-402.

Voss, A., Rothermund, K., \& Voss, J. (2004). Interpreting the parameters of the diffusion model : An empirical validation. Memory \& Cognition, 32(7), 1206-1220.

Wiecki, T. V, Sofer, I., \& Frank, M. J. (2013). HDDM : Hierarchical Bayesian estimation of the Drift-Diffusion Model in Python, 7(August), 1-10.

Winer, R. S. (1986). A Reference Price Model of Brand Choice for Frequently Purchased Products. Journal of Consumer Research, 13(2), 250-256.

Wright, G., \& Ayton, P. (1988). Decision time, subjective probability, and task difficulty. Memory \& Cognition, 16(2), 176-185. 